\title{
First-principles calculation of the Gilbert damping parameter via the linear response formalism with application to magnetic transition-metals and alloys
}

\author{
S. Mankovsky ${ }^{1}$, D. Ködderitzsch ${ }^{1}$, G. Woltersdorf ${ }^{2}$, and H. Ebert ${ }^{1}$ \\ 1 University of Munich, Department of Chemistry, \\ Butenandtstrasse 5-13, D-81377 Munich, Germany and \\ 2 Department of Physics, Universität Regensburg, 93040 Regensburg, Germany
}

( $\Omega$ Dated: September 9, 2018)

\begin{abstract}
A method for the calculations of the Gilbert damping parameter $\alpha$ is presented, which based on the linear response formalism, has been implemented within the fully relativistic Korringa-KohnRostoker band structure method in combination with the coherent potential approximation alloy theory. To account for thermal displacements of atoms as a scattering mechanism, an alloy-analogy model is introduced. This allows the determination of $\alpha$ for various types of materials, such as elemental magnetic systems and ordered magnetic compounds at finite temperature, as well as for disordered magnetic alloys at $T=0 \mathrm{~K}$ and above. The effects of spin-orbit coupling, chemical and temperature induced structural disorder are analyzed. Calculations have been performed for the $3 d$ transition-metals bcc Fe, hcp Co, and fcc Ni, their binary alloys bcc $\mathrm{Fe}_{1-x} \mathrm{Co}_{x}, \mathrm{fcc}_{\mathrm{Ni}_{1-x}} \mathrm{Fe}_{x}$, fcc $\mathrm{Ni}_{1-x} \mathrm{Co}_{x}$ and bcc $\mathrm{Fe}_{1-x} \mathrm{~V}_{x}$, and for $5 d$ impurities in transition-metal alloys. All results are in satisfying agreement with experiment.
\end{abstract}

PACS numbers: 72.25.Rb 71.20.Be 71.70.Ej 75.78.-n

\section{INTRODUCTION}

During the last decades dynamical magnetic properties have attracted a lot of interest due to their importance in the development of new devices for spintronics, in particular, concerning their miniaturization and fast time scale applications. A distinctive property of such devices is the magnetization relaxation rate characterizing the time scale on which a system being deviated from the equilibrium returns to it, or how fast the device can be switched from one state to another. In the case of dynamics of a uniform magnetization /vecM this property is associated with the Gilbert damping parameter $\tilde{G}(\mathbf{M})$ used first in the phenomenological Landau-Lifshitz (LL) ${ }^{1}$ and Landau-Lifshitz-Gilbert (LLG) theory ${ }^{2}$ describing the magnetization dynamics processes by means of the equation:

$$
\frac{1}{\gamma} \frac{d \mathbf{M}}{d \tau}=-\mathbf{M} \times \mathbf{H}_{\mathrm{eff}}+\mathbf{M} \times\left[\frac{\tilde{G}(\mathbf{M})}{\gamma^{2} M_{s}^{2}} \frac{d \mathbf{M}}{d \tau}\right]
$$

where $M_{s}$ is the saturation magnetization, $\gamma$ the gyromagnetic ratio and $\mathbf{H}_{\text {eff }}=-\partial_{\mathbf{M}} F[\mathbf{M}(\mathbf{r})]$ being the effective magnetic field. Sometimes it is more convenient to use a dimensionless Gilbert damping parameter $\alpha$ given by $\alpha=\tilde{G} /\left(\gamma M_{s}\right)$ (see, e.g. $\left.{ }^{3-5}\right)$. Safonov has generalized the Landau-Lifshitz equation by introducing a tensorial form for the Gilbert damping parameter with the diagonal terms characterising magnetization dissipation ${ }^{6}$. Being introduced as a phenomenological parameter, the Gilbert damping is normally deduced from experiment. In particular, it can be evaluated from the resonant line width in ferromagnetic-resonance (FMR) experiments. The difficulty of these measurements consists in the problem that there exist several different sources for the broadening of the line width, which have been discussed extensively in the literature ${ }^{7-13}$. The line width that is observed in ferromagnetic resonance spectra is usually caused by intrinsic and extrinsic relaxation effects. The extrinsic contributions are a consequence of spatially fluctuating magnetic properties due to sample imperfections. Short range fluctuations lead to two magnon scattering while long range fluctuations lead to an inhomogeneous line broadening due a superposition of local resonances ${ }^{14}$. In order to separate the intrinsic Gilbert damping from the extrinsic effects it is necessary to measure the frequency and angular dependence of the ferromagnetic resonance line width, e. g. two magnon scattering can be avoided when the magnetization is aligned along the film normal ${ }^{11}$ (perpendicular configuration). Usually one finds a linear frequency dependence with a zero frequency offset and one can write $\Delta H(\omega)=\alpha \frac{\omega}{\gamma}+\Delta H(0)$. When such measurements are performed over a wide frequency range the slope of $\Delta H$ as a function of frequency can be used to extract the intrinsic Gilbert damping constant. In metallic ferromagnets Gilbert damping is mostly caused by electron magnon scattering. In addition Gilbert-like damping can be caused by eddy currents. The magnitude of the eddy current damping is proportional to $d^{2}$, where $d$ is the sample thickness ${ }^{10}$. In sufficiently thin magnetic films $(d \leq 10 \mathrm{~nm})$ the eddy current damping can be neglected ${ }^{10}$. However, for very thin films relaxation mechanisms that occur at the interfaces can also increase and even dominate the damping. Such effects are spin pumping ${ }^{15,16}$ and the modified electronic structure at the interfaces. In the present work spin pumping and the modified interface electronic structure are not considered and we assume that bulk-like Gilbert damping dominates.

Much understanding of dynamical magnetic properties could in principle be obtained from the simulation of 
these processes utilizing time-dependent first-principles electronic structure calculations, that in turn would pave the way to developing and optimizing new materials for spintronic devices. In spite of the progress in the development of time-dependent density functional theory (TD-DFT) during the last decades ${ }^{17}$ that allows to study various dynamical processes in atoms and molecules from first principles, applications to solids are rare. This is due to a lack of universally applicable approximations to the exchange-correlation kernel of TD-DFT for solids. Thus, at the moment, a tractable approach consists in the use of the classical LLG equations, and employing parameters calculated within a microscopic approach. Note however that this approach can fail dealing with ultrafast magnetization dynamics, which is discussed, for instance, in Refs. [18 and 19], but is not considered in the present work.

Most of the investigations on the magnetization dissipation have been carried out within model studies. Here one has to mention, in particular, the so-called $s$ - $d$ or $p$ - $d$ exchange model ${ }^{20-23}$ based on a separate consideration of the localized 'magnetic' $d$-electrons and delocalized $s$ - and $p$-electrons mediating the exchange interactions between localized magnetic moments and responsible for the magnetization dissipation in the system. As was pointed out by Skadsem et al. ${ }^{24}$, the dissipation process in this case can be treated as an energy pumping out of the $d$-electron subsystem into the $s$-electron bath followed by its dissipation via spin-flip scattering processes. This model gave a rather transparent qualitative picture for the magnetization relaxation in diluted alloys, e.g. magnetic semiconductors such as GaMnAs. However, it fails to give quantitative agreement with experiment in the case of itinerant metallic systems (e.g. $3 d$-metal alloys), where the $d$-states are rather delocalised and strongly hybridized with the $s p$-electrons. As a consequence the treatment of all valence electrons on the same footing is needed, which leads to the requirement of first-principles calculations of the Gilbert damping going beyond a model-based evaluation.

Various such calculations of the Gilbert damping parameter are already present in the literature. They usually assume a certain dissipation mechanism, like Kambersky's breathing Fermi surface (BFS) ${ }^{25,26}$, or more general torque-correlation models $(\mathrm{TCM})^{3,27}$. These models include explicitly the spin-orbit coupling (SOC), highlighting its key role in the magnetization dissipation processes. However, the latter methods used for electronic structure calculations cannot take explicitly into account disorder in the system that in turn is responsible for the aforementioned spin-flip scattering process. Therefore, this has to be simulated by using external parameters characterizing the finite lifetime of the electronic states. This weak point was recently addressed by Brataas et al. ${ }^{4}$ who described the Gilbert damping by means of scattering theory. This development supplied the formal basis for the first parameter-free investigations on disordered alloys for which the dominant scattering mechanism is potential scattering caused by chemical disorder ${ }^{5}$.

Theoretical investigations of the magnetization dissipation by means of first-principles calculations of the Gilbert damping parameter already brought much understanding of the physical mechanisms responsible for this effect. First of all, key roles are played by two effects: the SOC of the atomic species contained in the system and scattering on various imperfections, either impurities or structural defects, phonons, etc. Accounting for the crucial role of scattering processes responsible for the energy dissipation, different types of scattering phenomena have to be considered. One can distinguish between the ordered-compound or pure-element systems for which electron-phonon scattering is a very important mechanism for relaxation, and disordered alloys with dominating scattering processes resulting from randomly distributed atoms of different types. In the first case, the Gilbert damping behavior is rather different at low and high temperatures. At high temperature atomic displacements create random potentials leading to SOC-induced spin-flip scattering. At low temperature, where the magnetization dissipation is well described via the BFS (Breathing Fermi-surface) mechanism ${ }^{25,26}$, the spin-conserving electron-phonon scattering is required to bring the electronic subsystem to the equilibrium at every step of the magnetization rotation, i.e. to reoccupy the modified electronic states.

In this contribution we describe a formalism for the calculation of the Gilbert damping equivalent to that of Brataas et $a l .^{4}$, however, based on the linear responce theory ${ }^{28}$ as implemented in fully relativistic multiple scattering based Korringa-Kohn-Rostoker (KKR) formalism. It will be demonstrated that this allows to treat elegantly and efficiently the temperature dependence of $\alpha$ in pure crystals as well as disordered alloys.

\section{THEORETICAL APPROACH}

To have direct access to real materials and to obtain a deeper understanding of the origin of the properties observed experimentally, the phenomenological Gilbert damping parameter has to be treated on a microscopic level. This implies to deal with the electrons responsible for the energy dissipation in the magnetic dynamical processes. Thus, one equates the corresponding expressions for the dissipation rate obtained in the phenomenological and microscopic approaches $\dot{E}_{\text {mag }}=\dot{E}_{\text {dis }}$. Although a temporal variation of the magnetization is a required condition for the energy dissipation to occur, the Gilbert damping parameter is defined in the limit $\omega \rightarrow 0$ (see e.g., Ref. [24]) and therefore can be calculated within the adiabatic approximation.

In the phenomenological LLG theory the time dependent magnetization $\mathbf{M}(t)$ is described by Eq. (1). Accordingly, the time derivative of the magnetic energy is 
given by:

$$
\dot{E}_{\mathrm{mag}}=\mathbf{H}_{\mathrm{eff}} \cdot \frac{d \mathbf{M}}{d \tau}=\frac{1}{\gamma^{2}}(\dot{\hat{\mathbf{m}}})^{T}[\tilde{G}(\mathbf{M}) \dot{\hat{\mathbf{m}}}]
$$

where $\hat{\mathbf{m}}=\mathbf{M} / M_{s}$ denotes the normalized magnetization. To represent the Gilbert damping parameter in terms of a microscopic theory, following Brataas et al. ${ }^{4}$, the energy dissipation is associated with the electronic subsystem. The dissipation rate upon the motion of the magnetization $\dot{E}_{\mathrm{dis}}=\left\langle\frac{d \hat{H}}{d \tau}\right\rangle$, is determined by the underlying Hamiltonian $\hat{H}(\tau)$. Assuming a small deviation of the magnetic moment from the equilibrium the normalized magnetization $\hat{\mathbf{m}}(\tau)$ can be expanded around the equilibrium magnetization $\hat{\mathbf{m}}_{0}$

$$
\hat{\mathbf{m}}(\tau)=\hat{\mathbf{m}}_{0}+\mathbf{u}(\tau),
$$

resulting in the expression for the linearized time dependent Hamiltonian for the system brought out of equilibrium:

$$
\hat{H}=\hat{H}_{0}\left(\hat{\mathbf{m}}_{0}\right)+\sum_{\mu} u_{\mu} \frac{\partial}{\partial u_{\mu}} \hat{H}\left(\hat{\mathbf{m}}_{0}\right) .
$$

Due to the small deviation from the equilibrium, $\dot{E}_{\text {dis }}$ can be obtained within the linear response formalism, leading to the expression ${ }^{4}$ :

$$
\begin{gathered}
\dot{E}_{\mathrm{dis}}=-\pi \hbar \sum_{i j} \sum_{\mu \nu} \dot{u}_{\mu} \dot{u}_{\nu}\left\langle\psi_{i}\left|\frac{\partial \hat{H}}{\partial u_{\mu}}\right| \psi_{j}\right\rangle\left\langle\psi_{j}\left|\frac{\partial \hat{H}}{\partial u_{\nu}}\right| \psi_{i}\right\rangle \\
\times \delta\left(E_{F}-E_{i}\right) \delta\left(E_{F}-E_{j}\right)
\end{gathered}
$$

where $E_{F}$ is the Fermi energy and the sums run over all eigenstates of the system. As Eq. (5) characterizes the rate of the energy dissipation upon transition of the system from the tilted state to the equilibrium, it can be identified with the corresponding phenomenological quantity in Eq. (2), $\dot{E}_{\text {mag }}=\dot{E}_{\text {dis }}$. This leads to an explicit expression for the Gilbert damping tensor $\tilde{G}$ or equivalently for the damping parameter $\alpha=\tilde{G} /\left(\gamma M_{s}\right)$ (Ref. [4]):

$$
\begin{gathered}
\alpha_{\mu \nu}=-\frac{\hbar \gamma}{\pi M_{s}} \sum_{i j} \sum_{\mu \nu}\left\langle\psi_{i}\left|\frac{\partial \hat{H}}{\partial u_{\mu}}\right| \psi_{j}\right\rangle\left\langle\psi_{j}\left|\frac{\partial \hat{H}}{\partial u_{\nu}}\right| \psi_{i}\right\rangle \\
\times \delta\left(E_{F}-E_{i}\right) \delta\left(E_{F}-E_{j}\right)
\end{gathered}
$$

where the summation is running over all states at the Fermi surface $E_{F}$.

In full analogy to the problem of electric conductivity $^{29}$, the sum over eigenstates $\left|\psi_{i}\right\rangle$ may be expressed in terms of the retarded single-particle Green's function $\operatorname{Im} G^{+}\left(E_{F}\right)=-\pi \sum_{i}\left|\psi_{i}\right\rangle\left\langle\psi_{i}\right| \delta\left(E_{F}-E_{i}\right)$. This leads for the parameter $\alpha$ to a Kubo-Greenwood-like equation:

$$
\begin{aligned}
\alpha_{\mu \nu}= & -\frac{\hbar \gamma}{\pi M_{s}} \operatorname{Trace} \\
& \left\langle\frac{\partial \hat{H}}{\partial u_{\mu}} \operatorname{Im} G^{+}\left(E_{F}\right) \frac{\partial \hat{H}}{\partial u_{\nu}} \operatorname{Im} G^{+}\left(E_{F}\right)\right\rangle_{c}
\end{aligned}
$$

with $\langle\ldots\rangle_{c}$ indicating a configurational average in case of a disordered system.

The most reliable way to account for spin-orbit coupling as the source of Gilbert damping is to evaluate Eq. (7) using a fully relativistic Hamiltonian within the framework of local spin density formalism (LSDA) ${ }^{30}$ :

$$
\hat{H}=c \boldsymbol{\alpha} \cdot \mathbf{p}+\beta m c^{2}+V(\mathbf{r})+\beta \boldsymbol{\sigma} \cdot \hat{\mathbf{m}} B(\mathbf{r}) .
$$

Here $\alpha_{i}$ and $\beta$ are the standard Dirac matrices, $\boldsymbol{\sigma}$ denotes the vector of relativistic Pauli matrices, and $\mathbf{p}$ is the relativistic momentum operator ${ }^{31}$. The functions $V(\mathbf{r})$ and $\mathbf{B}=\boldsymbol{\sigma} \cdot \hat{\mathbf{m}} B(\mathbf{r})$ are the spin-averaged and spin-dependent parts, respectively, of the LSDA potential. The spin density $\mathbf{m}_{s}(\mathbf{r})$ as well as the effective exchange field $\mathbf{B}(\mathbf{r})$ are assummed to be collinear within the unit cell and aligned along the $z$-direction in the equilibrium (i. e. $\mathbf{m}_{s, 0}(\mathbf{r})=m_{s}(\mathbf{r}) \hat{\mathbf{m}}_{0}=m_{s}(\mathbf{r}) \mathbf{e}_{z}$ and $\left.\mathbf{B}_{0}(\mathbf{r})=B(\mathbf{r}) \hat{\mathbf{m}}_{0}=B(\mathbf{r}) \mathbf{e}_{z}\right)$. Tilting of the magnetization direction by the angle $\theta$ according to Eq. (3), i.e. $\mathbf{m}_{s}(\mathbf{r})=m_{s}(\mathbf{r}) \hat{\mathbf{m}}=m_{s}(\mathbf{r})(\sin \theta \cos \phi, \sin \theta \sin \phi, \cos \theta)$ and $\mathbf{B}(\mathbf{r})=B(\mathbf{r}) \hat{\mathbf{m}}$ leads to a perturbation term in the Hamiltonian

$$
\Delta V(r)=\beta \boldsymbol{\sigma} \cdot\left(\hat{\mathbf{m}}-\hat{\mathbf{m}}_{0}\right) B(\mathbf{r})=\beta \boldsymbol{\sigma} \cdot \mathbf{u} B(\mathbf{r}),
$$

with (see Eq. (4))

$$
\frac{\partial}{\partial u_{\mu}} \hat{H}\left(\hat{\mathbf{m}}_{0}\right)=\beta \sigma_{\mu} B(\mathbf{r}) .
$$

The Green's function $G^{+}$in Eq. (7) can be obtained in a very efficient way by using the spin-polarized relativistic version of multiple scattering theory ${ }^{30}$ that allows us to treat magnetic solids:

$$
\begin{array}{r}
G^{+}\left(\mathbf{r}, \mathbf{r}^{\prime}, E\right)=\sum_{\Lambda \Lambda^{\prime}} Z_{\Lambda}^{n}(\mathbf{r}, E) \tau_{\Lambda \Lambda^{\prime}}^{n m}(E) Z_{\Lambda^{\prime}}^{m \times}\left(\mathbf{r}^{\prime}, E\right) \\
-\delta_{n m} \sum_{\Lambda}\left[Z_{\Lambda}^{n}(\mathbf{r}, E) J_{\Lambda^{\prime}}^{n \times}\left(\mathbf{r}^{\prime}, E\right) \Theta\left(r_{n}^{\prime}-r_{n}\right)\right. \\
\left.+J_{\Lambda}^{n}(\mathbf{r}, E) Z_{\Lambda^{\prime}}^{n \times}\left(\mathbf{r}^{\prime}, E\right) \Theta\left(r_{n}-r_{n}^{\prime}\right)\right]
\end{array}
$$

Here $\mathbf{r}, \mathbf{r}^{\prime}$ refer to site $n$ and $m$, respectively, where $Z_{\Lambda}^{n}(\mathbf{r}, E)=Z_{\Lambda}\left(\mathbf{r}_{n}, E\right)=Z_{\Lambda}\left(\mathbf{r}-\mathbf{R}_{n}, E\right)$ is a function centered at site $\mathbf{R}_{n}$. The four-component wave functions $Z_{\Lambda}^{n}(\mathbf{r}, E)\left(J_{\Lambda}^{n}(\mathbf{r}, E)\right)$ are regular (irregular) solutions to the single-site Dirac equation labeled by the combined quantum numbers $\Lambda(\Lambda=(\kappa, \mu))$, with $\kappa$ and $\mu$ being the spin-orbit and magnetic quantum numbers ${ }^{31}$. The superscript $\times$ indicates the left hand side solution of the Dirac equation. $\tau_{\Lambda \Lambda^{\prime}}^{n m}(E)$ is the so-called scattering path operator that transfers an electronic wave coming in at site $m$ into a wave going out from site $n$ with all possible intermediate scattering events accounted for.

Using matrix notation with respect to $\Lambda$, this leads to the following expression for the damping parameter:

$$
\alpha_{\mu \mu}=\frac{g}{\pi \mu_{\text {tot }}} \sum_{n} \operatorname{Trace}\left\langle\underline{T}^{0 \mu} \underline{\tilde{\tau}}^{0 n} \underline{T}^{n \mu} \underline{\tilde{\tau}}^{n 0}\right\rangle_{c}
$$

with the g-factor $2\left(1+\mu_{\text {orb }} / \mu_{\text {spin }}\right)$ in terms of the spin and orbital moments, $\mu_{\text {spin }}$ and $\mu_{\text {orb }}$, respectively, the 
total magnetic moment $\mu_{\text {tot }}=\mu_{\text {spin }}+\mu_{\text {orb }}, \tilde{\tau}_{\Lambda \Lambda^{\prime}}^{0 n}=$ $\frac{1}{2 i}\left(\tau_{\Lambda \Lambda^{\prime}}^{0 n}-\tau_{\Lambda^{\prime} \Lambda}^{0 n}\right)$ and with the energy argument $E_{F}$ omitted. The matrix elements in Eq. (12) are identical to those occurring in the context of exchange coupling ${ }^{32}$ :

$$
\begin{aligned}
T_{\Lambda^{\prime} \Lambda}^{n \mu} & =\int d^{3} r Z_{\Lambda^{\prime}}^{n \times}(\mathbf{r})\left[\frac{\partial}{\partial u_{\mu}} \hat{H}\left(\hat{\mathbf{m}}_{0}\right)\right] Z_{\Lambda}^{n}(\mathbf{r}) \\
& =\int d^{3} r Z_{\Lambda^{\prime}}^{n \times}(\mathbf{r})\left[\beta \sigma_{\mu} B_{x c}(\mathbf{r})\right] Z_{\Lambda}^{n}(\mathbf{r}) .
\end{aligned}
$$

The expression in Eq. (12) for the Gilbert-damping parameter $\alpha$ is essentially equivalent to the one obtained within the torque correlation method (see e.g. Refs. [3335]). However, in contrast to the conventional TCM the electronic structure is here represented using the retarded electronic Green function giving the present approach much more flexibility. In particular, it does not rely on a phenomenological relaxation time parameter.

The expression Eq. (12) can be applied straightforwardly to disordered alloys. This can be done by describing in a first step the underlying electronic structure (for $T=0 \mathrm{~K}$ ) on the basis of the coherent potential approximation (CPA) alloy theory. In the next step the configurational average in Eq. (12) is taken following the scheme worked out by Butler ${ }^{29}$ when dealing with the electrical conductivity at $T=0 \mathrm{~K}$ or residual resistivity, respectively, of disordered alloys. This implies in particular that so-called vertex corrections of the type $\left\langle T_{\mu} \operatorname{Im} G^{+} T_{\nu} \operatorname{Im} G^{+}\right\rangle_{c}-\left\langle T_{\mu} \operatorname{Im} G^{+}\right\rangle_{c}\left\langle T_{\nu} \operatorname{Im} G^{+}\right\rangle_{c}$ that account for scattering-in processes in the language of the Boltzmann transport formalism are properly accounted for.

One has to note that the factor $\frac{g}{\mu_{t o t}}$ in Eq. (12) is separated from the configurational average $\langle\ldots\rangle_{c}$, although both values, $g$ and $\mu_{t o t}$, have to represent the average per unit cell doing the calculations for compounds and alloys. This approximation is rather reasonable in the case of compounds or alloys where the properties of the elements of the system are similar (e.g. 3d-element alloys), but can be questionable in the case of systems containing elements exhibiting significant differences $(3 d-5 d-, 3 d-4 f$ compounds, etc), or in the case of non-uniform systems as discussed by Nibarger et $\mathrm{al}^{36}$.

Thermal vibrations as a source of electron scattering can in principle be accounted for by a generalization of Eqs. (7) - (13) to finite temperatures and by including the electron-phonon self-energy $\Sigma_{e l-p h}$ when calculating the Green's function $G^{+}$. Here we restrict our consideration to elastic scattering processes by using a quasi-static representation of the thermal displacements of the atoms from their equilibrium positions. The atom displaced from the equilibrium position in the lattice results in a corresponding variation $\Delta \underline{t}^{n}=\underline{t}^{n}-\underline{t}_{0}^{n}$ of the single-site scattering matrix in the global frame of reference ${ }^{37,38}$. A single-site scattering matrix $\underline{t}^{n}$ (the underline denotes a matrix in an angular momentum representation $\Lambda$ ) for the atom $n$ displaced by the value $\mathbf{s}_{\nu}^{n}$ from the equilibrium position in the lattice can be obtained using the transformation matrices ${ }^{37,39}$

$$
\begin{aligned}
U_{L L^{\prime}}^{n}\left(\mathbf{s}_{\nu}, E\right) & =4 \pi \sum_{L^{\prime \prime}} i^{l^{\prime \prime}+l-l^{\prime}} \\
& \times C_{L L^{\prime} L^{\prime \prime}} j_{l^{\prime \prime}}\left(s_{\nu}^{n} \sqrt{E}\right) Y_{L^{\prime \prime}}\left(\hat{\mathbf{s}}_{\nu}^{n}\right) .
\end{aligned}
$$

Here $m_{e}$ is the electron mass, $j_{l}$ a spherical Bessel function, $C_{L L^{\prime} L^{\prime \prime}}$ stands for the Gaunt coefficients, and a non-relativistic angular momentum representation with $L=\left(l, m_{l}\right)$ has been used. Performing a Clebsch-Gordon transformation for the transformation matrix $U_{L L^{\prime}}^{n}$ to the relativistic $\Lambda$ representation, the $t$ matrix $\underline{t}^{n}$ for the shifted atom can be obtained from the non-shifted one $\underline{t}_{0}^{n}$ from the expression

$$
\underline{t}_{\nu}^{n}=\left(\underline{U}_{\nu}^{n}\right)^{-1} \underline{t}_{0}^{n} \underline{U}_{\nu}^{n}
$$

Treating for a discrete set of displacements $\mathbf{s}_{\nu}^{n}$ each displacement as an alloy component, we introduce an alloy-analogy model to average over the set $\mathbf{s}_{\nu}^{n}$ that is chosen to reproduce the thermal root mean square average displacement $\sqrt{\left\langle u^{2}\right\rangle_{T}}$ for a given temperature $T$. This in turn may be set according to $\left\langle u^{2}\right\rangle_{T}=$ $\frac{1}{4} \frac{3 h^{2}}{\pi^{2} m k \Theta_{D}}\left[\frac{\Phi\left(\Theta_{D} / T\right)}{\Theta_{D} / T}+\frac{1}{4}\right]$ with $\Phi\left(\Theta_{D} / T\right)$ the Debye function, $h$ the Planck constant, $k$ the Boltzmann constant and $\Theta_{D}$ the Debye temperature ${ }^{40}$. Ignoring the zero temperature term $1 / 4$ and assuming a frozen potential for the atoms, the situation can be dealt with in full analogy to the treatment of disordered alloys on the basis of the CPA (see above).

For small displacements the transformation Eq. (14) can be expanded with respect to $\mathbf{s}_{\nu}^{n}$ (see Ref. [39]) resulting in a linear dependence on $\mathbf{s}_{\nu}^{n}$ for non-vanishing contributions with $\Delta l=\left|l-l^{\prime}\right|= \pm 1$. This leads, in particular, in the presence of atomic displacements for transition-metals (TM), for which an angular momentum cut-off of $l_{\max }=2$ in the KKR multiple scattering expansion is in general sufficient for an undistorted lattice, to an angular momentum expansion up to at least $l_{\max }=3$. However, this is correct only under the assumption of very small displacements allowing linearisation of the transformation $U$ with respect to the displacement amplitude $s$. Thus, since the temperature increase leads to a monotonous increase of $s$, the cut-off $l_{\max }$ should also be increased.

\section{MODEL CALCULATIONS}

In the following we present results of calculations for which single parameters have artificially been manipulated in the first-principles calculations in order to systematically reveal their role for the Gilbert-damping. This approach is used to disentangle competing influences on the Gilbert-damping parameter. 


\section{A. Vertex corrections}

The impact of vertex corrections is shown in Fig. 1 for two different cases: Fig. 1(a) represents the Gilbert damping parameter for an $\mathrm{Fe}_{1-x} \mathrm{~V}_{x}$ disordered alloy as a function of concentration, while Fig. 1(b) gives the corresponding value for pure Fe in the presence of temperature induced disorder and plotted as a function of temperature. Both figures show results calculated with and without vertex corrections allowing for comparison. First of all, a significant effect of the vertex corrections is noticeable in both cases, although the variation depends on increasing concentration of $\mathrm{V}$ in the binary $\mathrm{Fe}_{1-x} \mathrm{~V}_{x}$ alloy and the temperature in the case of pure Fe, respectively. Some differences in their behavior can be explained by the differences of the systems under consideration. Dealing with temperature effects via the alloy analogy model, the system is considered as an effective alloy consisting of a fixed number of components characterizing different types of displacements. Thus, in this case the temperature effect is associated with the increase of disorder in the system caused only by the increase of the displacement amplitude, or, in other words - with the strength of scattering potential experienced by the electrons represented by $\underline{t}^{n}(T)-\underline{t}_{0}^{n}$. In the case of a random alloy the $A_{1-x} B_{x}$ variation of the scattering potential, as well as the difference $\underline{t}_{A}^{n}-\underline{t}_{B}^{n}$, upon changing the concentrations is less pronounced for small amounts of impurities $B$ and the concentration dependence is determined by the amount of scatterers of different types. However, when the concentration of impurities increases, the potentials of the components are also modified (this is reflected, e.g., in the shift of electronic states with respect to the Fermi level, that will be discussed below) and this can lead to a change of the concentration dependence of the vertex corrections. An important issue which one has to stress that neglect of the vertex corrections may lead to the unphysical result, $\alpha<0$, as is shown in Fig. 1(a). In terms of the Boltzmann transport formalism, this is because of the neglect of the scattering-in term ${ }^{41}$ leading obviously to an incomplete description of the energy transfer processes.

\section{B. Influence of spin-orbit coupling}

As was already discussed above, the spin-orbit coupling for the electrons of the atoms composing the system is the main driving force for the magnetization relaxation, resulting in the energy transfer from the magnetic subsystem to the crystal lattice. Thus, the Gilbert damping parameter should approach zero upon decreasing the SOC in the system. Fig. 2 shows the results for $\mathrm{Py}+15 \% \mathrm{Os}$, where $\sqrt{\alpha}$ is plotted as a function of the scaling parameter of the spin-orbit coupling ${ }^{42}$ applied to all atoms in the alloy. As one can see, $\sqrt{\alpha}$ has a nearly linear dependence on SOC implying that $\alpha$ varies in second order in the strength of the spin-orbit coupling ${ }^{43}$.

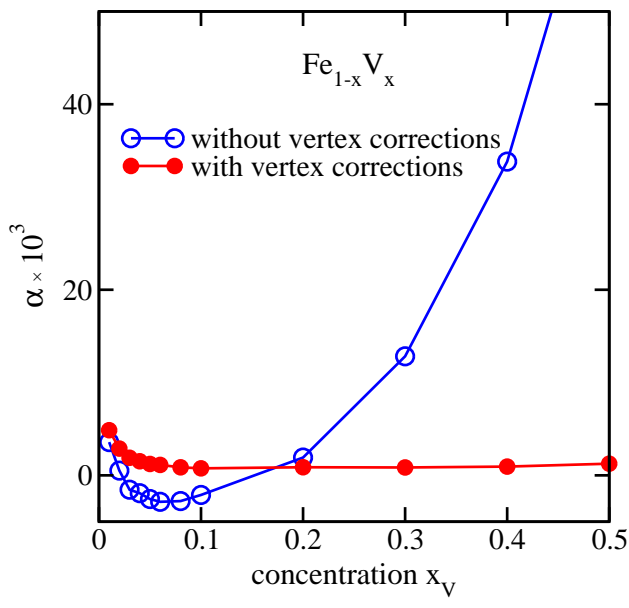

(a)

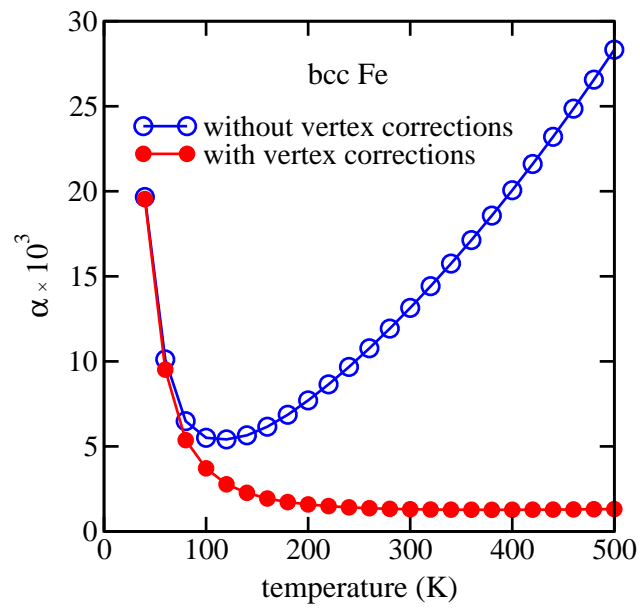

(b)

FIG. 1. The Gilbert damping parameter for (a) bcc $\mathrm{Fe}_{1-x} \mathrm{~V}_{x}$ $(T=0 \mathrm{~K})$ as a function of $\mathrm{V}$ concentration and (b) for bcc-Fe as a function of temperature. Full (open) symbols give results with (without) the vertex corrections.

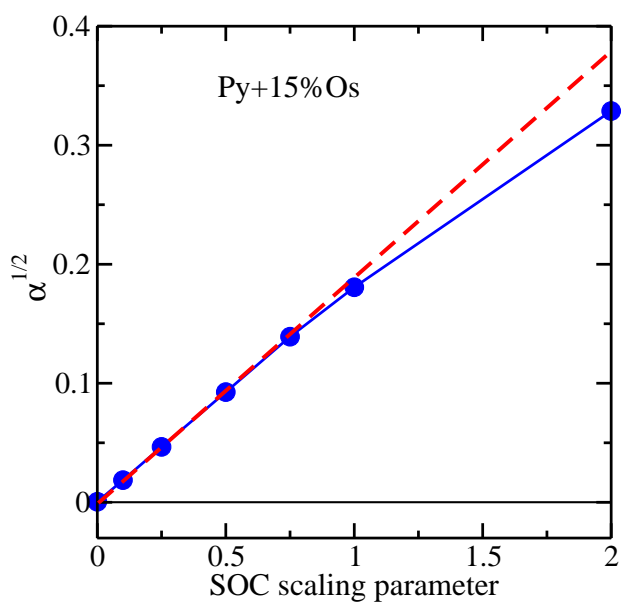

FIG. 2. The Gilbert damping parameter for Py $+15 \%$ Os as a function of the scaling parameter of spin-orbit coupling applied to all atoms contained in the alloy. Red dashed line in plot - linear fit. The values 0 and 1 for the SOC scaling parameter correspond to the scalar-relativistic Schrödinger-like and fully relativistic Dirac equations, respectively. 


\section{RESULTS AND DISCUSSIONS}

\section{A. $3 d$ transition-metals}

We have mentioned above the crucial role of scattering processes for the energy dissipation in magnetization dynamic processes. In pure metals, in the absence of any impurity, the electron-phonon scattering mechanism is of great importance, although it plays a different role in the low- and high-temperature regimes. This was demonstrated by Ebert et al. ${ }^{28}$ using the alloy analogy approach, as well as by $\mathrm{Liu}^{44}$ et al. using the 'frozen thermal lattice disorder' approach. In fact both approaches are based on the quasi-static treatment of thermal displacements. However, while the average is taken by the CPA within the alloy analogy model the latter requires a sequence of super-cell calculations for this purpose.

As a first example bcc Fe is considered here. The calculations have been performed accounting for the temperature induced atomic displacements from their equilibrium positions, according to the alloy analogy scheme described in section II. This leads, even for pure systems, to a scattering process and in this way to a finite value for $\alpha$ (see Fig. 3(a)). One can see that the experimental results available in the literature are rather different, depending on the conditions of the experiment. In particular, the experimental results Expt. 2 (Ref. [45]) and Expt. 3 (Ref. [46]) correspond to bulk while the measurements Expt. 1 (Ref. [47]) have been done for an ultrathin film with $2.3 \mathrm{~nm}$ thickness. The Gilbert damping constant obtained within the present calculations for bcc Fe (circles, $a=5.44$ a.u.) is compared in Fig. 3(a) with the experiment exhibiting rather good agreement at the temperature above $100 \mathrm{~K}$ despite a certain underestimation. One can also see a rather pronounced increase of the Gilbert damping observed in the experiment above 400 K (Fig. 3(a), Expt. 2 and Expt. 3), while the theoretical value shows only little temperature dependent behavior. Nevertheless, the increase of the Gilbert damping with temperature becomes more pronounced when the temperature induced lattice expansion is taken into account, that can be seen from the results obtained for $a=5.45$ a.u. (squares). Thick lines are used to stress the temperature regions for which corresponding lattice parameters are more appropriate. At low temperatures, below $100 \mathrm{~K}$, the calculated Gilbert damping parameter goes up when the temperature decreases, that was observed only in the recent experiment ${ }^{47}$. This behavior is commonly denoted as a transition from low-temperature conductivity-like to high-temperature resistivity-like behavior reflecting the dominance of intra- and inter-band transitions, respectively ${ }^{3}$. The latter are related to the increase of the smearing of electron energy bands caused by the increase of scattering events with temperature. Note that even a small amount of impurities reduces strongly the conductivity-like behavior ${ }^{28,45}$, leading to the more pronounced effect of impurity-scattering processes due to the increase of scattering events caused by chemical disorder. Large discrepancies between the latter experimental data ${ }^{47}$ and theoretical results of the $\alpha$ calculations for bcc Fe are related to the very small thickness of the film investigated experimentally, that leads to an increase of spin-transfer channels for magnetization dissipation as was discussed above.

Results for the temperature dependent Gilbertdamping parameter $\alpha$ for hcp Co are presented in Fig. 3(b) which shows, despite certain underestimation, a reasonable agreement with the experimental results ${ }^{45}$. The general trends at low and high temperatures are similar to those seen in Fe.

The results for pure Ni are given in Fig. 3(c) that show in full accordance with experiment a rapid decrease of $\alpha$ with increasing temperature until a regime with a weak variation of $\alpha$ with $T$ is reached.

Note that in the discussions above we have treated $\alpha$ as a scalar instead of a tensorial quantity ignoring a possible anisotropy of the damping processes. This approximation is reasonable for the systems considered above with the magnetization directions along a three- or fourfold symmetry axis (see, e.g., the discussions in Ref. [48 and 49]). For a more detailed discussion of this issue the anisotropy of the Gilbert damping tensor $\underline{\underline{\alpha}}(\mathbf{M})$ has been investigated for bcc Fe. To demonstrate the dependence of $\underline{\alpha}$ on the magnetization direction $\mathbf{M}$, the calculations $\overline{\bar{h}}$ ave been performed for $\mathbf{M}=\hat{z}|\mathbf{M}|$ with the $\hat{z}$ axis taken along the $\langle 001\rangle,\langle 111\rangle$ and $\langle 011\rangle$ crystallographic directions. Fig. 4 presents the temperature dependence of the diagonal elements $\alpha_{x x}$ and $\alpha_{y y}$. As to be expected for symmetry reasons, $\alpha_{x x}$ differs from $\alpha_{y y}$ only in the case of $\hat{z} \|\langle 011\rangle$. One can see that the anisotropic behavior of the Gilbert damping is pronounced at low temperatures. With an increase of the temperature the anisotropy nearly disappears, because of the smearing of the energy bands caused by thermal vibrations ${ }^{49}$. A similar behavior is caused by impurities with a random distribution, as was observed for example for the $\mathrm{Fe}_{0.95} \mathrm{Si}_{0.05}$ alloy system. The calculations of the diagonal elements $\alpha_{x x}$ and $\alpha_{y y}$ for two different magnetization directions along $\langle 001\rangle$ and $\langle 011\rangle$ axes give $\alpha_{x x}=\alpha_{y y}=0.00123$ in the first case and $\alpha_{x x}=0.00123$ and $\alpha_{y y}=0.00127$ in the second, i.e. the damping is nearly isotropic.

The damping parameter $\alpha$ increases very rapidly with decreasing temperature in the low temperature regime $(T \leq 100 \mathrm{~K})$ for all pure ferromagnetic $3 d$ metals, Fe, Co, and Ni (see Fig. 3), leading to a significant discrepancy between theoretical and experimental results in this regime. The observed discrepancy between theory and experiment can be related to the exact limit $\omega=0$ taken in the expression for the Gilbert damping parameter. Korenmann and Prange ${ }^{13}$ have analyzed the magnon damping in the limit of small wave vector of magnons $\mathbf{q} \rightarrow 0$, assuming indirect transitions in the electron subsystem and taking into account the finite lifetime $\tau$ of the Bloch states due to electron-phonon scattering. They discuss the limiting cases of low and high temperatures showing the analogy of the present problem with the problem of 

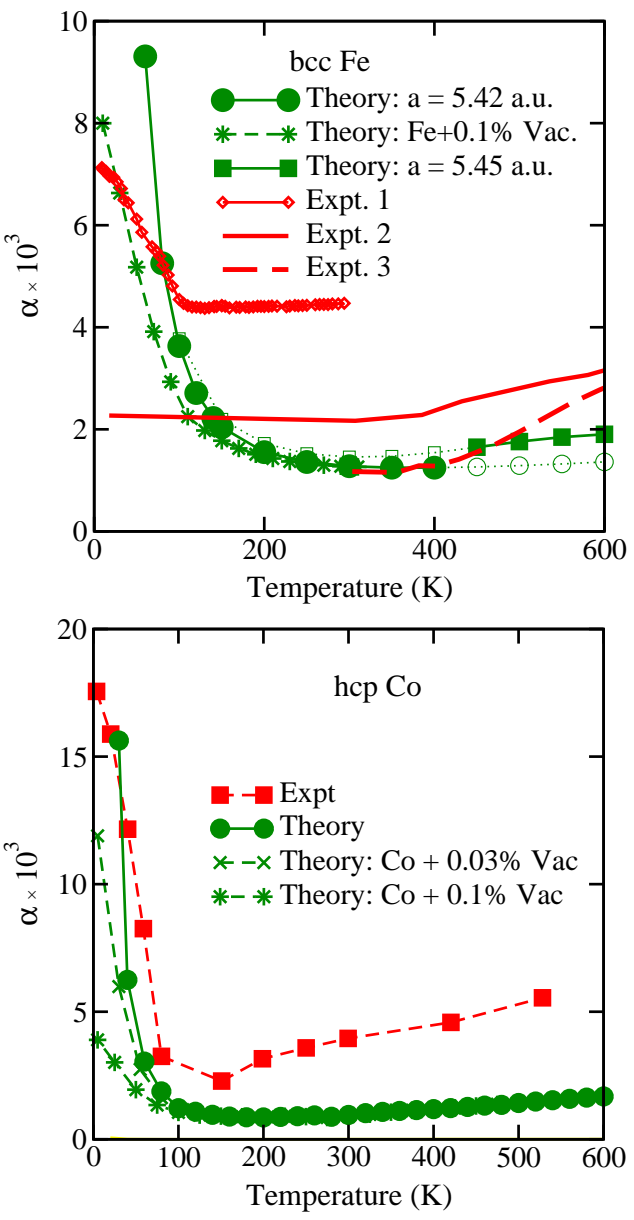

(a)

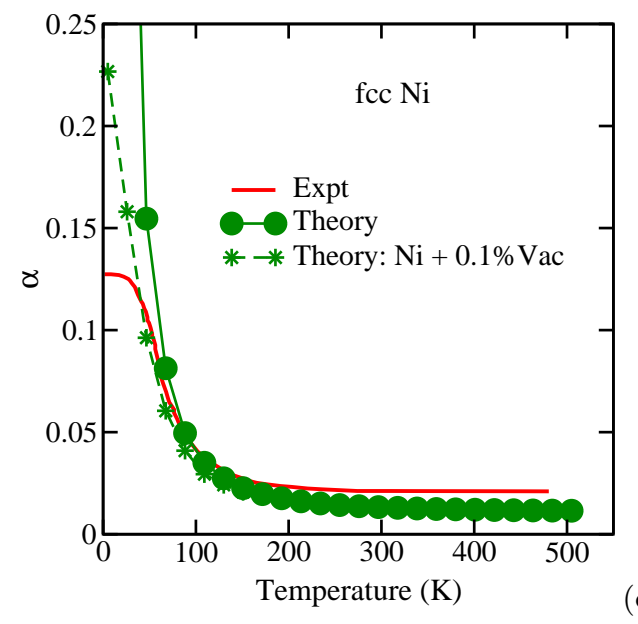

(b)

(c)

FIG. 3. Temperature variation of the Gilbert damping parameter of pure systems. Comparison of theoretical results with experiment: (a) bcc-Fe: circles and squares show the results for ideal bcc Fe for two lattice parameters, $a=5.42$ a.u. and $a=5.45$ a.u.; stars show theoretical results for bcc Fe ( $a=5.42$ a.u.) with $0.1 \%$ of vacancies (Expt. 1 - Ref. [47], Expt. 2 - Ref. [45], Expt. 3 - Ref. [46]); (b) hcp-Co: circles show theoretical results for ideal hcp Co, stars - for Co with $0.03 \%$ of vacancies, and 'pluses' - for Co with $0.1 \%$ of vacancies (Expt. Ref. [45]); and (c) fcc-Ni (Expt. Ref. [45]). extreme cases for the conductivity leading to the normal and anomalous skin effect. On the basis of their result, the authors point out that the expression for the Gilbert damping obtained by Kambersky ${ }^{25}$, with $\alpha \sim \tau$ is correct in the limit of small lifetime (i.e. $q v_{F} \tau \ll 1$, in their model consideration, where $q$ is a magnon wave vector and $v_{F}$ is a Fermi velocity of the electron). In the lowtemperature limit the lifetime $\tau$ increases with decreasing $T$ and one has to use the expression corresponding to the 'anomalous' skin effect for the conductivity, i.e. $\alpha \sim \tan ^{-1}\left(q v_{F} \tau\right) / q v_{F}$, leading to a saturation of $\alpha$ upon the increase of $\tau$.

Another possible reason for the low-temperature behavior of the Gilbert damping observed experimentally can be structural defects present in the material. To simulate this effect, calculations have been performed for fcc $\mathrm{Ni}$ and bcc Fe with $0.1 \%$ of vacancies and for hcp Co with $0.1 \%$ and $0.03 \%$ of vacancies. Fig. 3(a)-(c) shows the corresponding temperature dependence of the Gilbert damping parameter approaching a finite value for $T \rightarrow 0$. The remaining difference in the $T$-dependent behavior can be attributed to the non-linear dependence of the scattering cross section at low temperatures as is discussed in the literature for transport properties of metals and is not accounted for within the present approximation.

\section{B. $3 d$ Transition-metal alloys}

As is mentioned above, the use of the linear response formalism within multiple scattering theory for the electronic structure calculations allows us to perform the necessary configurational averaging in a very efficient way avoiding supercell calculations and to study with moderate effort the influence of varying alloy composition on $\alpha$. The corresponding approach has been applied to

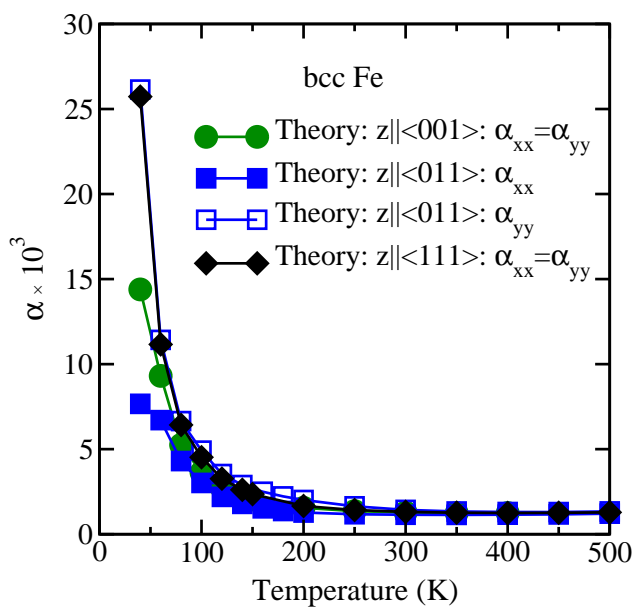

FIG. 4. Temperature variation of the $\alpha_{x x}$ and $\alpha_{y y}$ components of the Gilbert damping tensor of bcc Fe with the magnetization direction taken along different crystallographic directions: $\mathbf{M}=\hat{z}|\mathbf{M}| \|\langle 001\rangle$ (circles), $\mathbf{M} \|\langle 011\rangle$ (squares), $\mathbf{M} \|\langle 111\rangle$ (diamonds). 

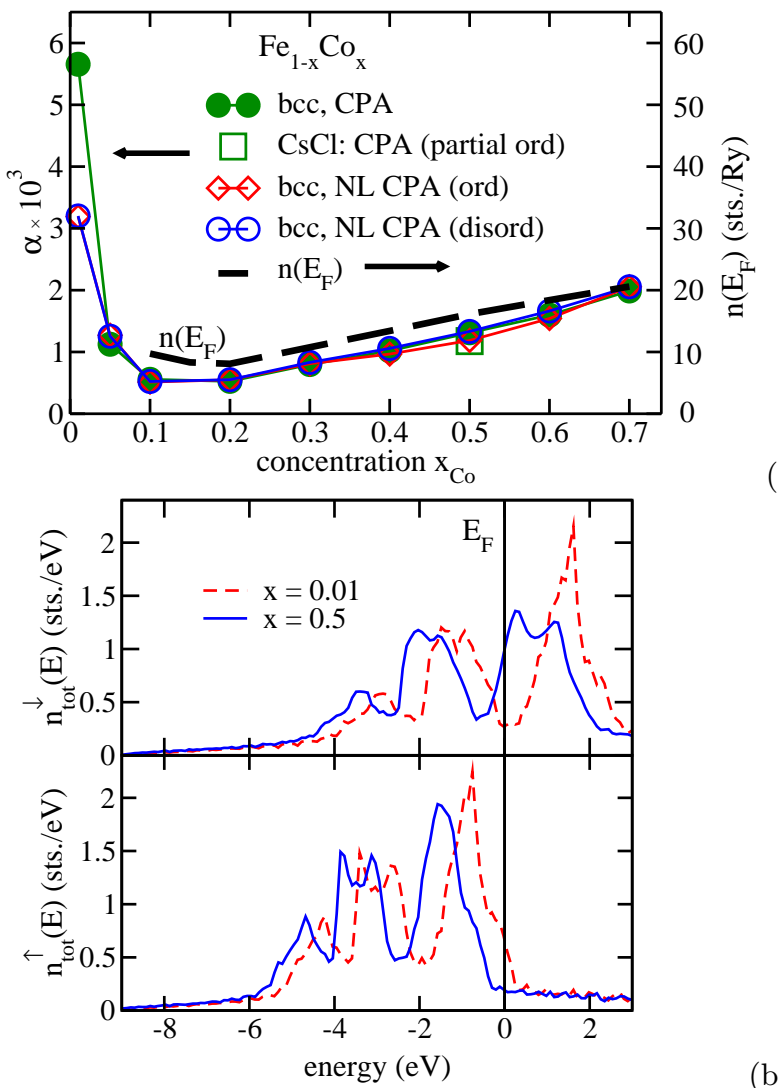

(a)

FIG. 5. (a) Theoretical results for the Gilbert damping parameter of bcc $\mathrm{Fe}_{1-x} \mathrm{Co}_{x}$ as a function of Co concentration: CPA results for the bcc structure (full circles) describing the random alloy system, results for the partially ordered system (opened square) for $x=0.5$ (i.e. for $\mathrm{Fe}_{1-x} \mathrm{Co}_{x}$ alloy with $\mathrm{CsCl}$ structure and alloy components randomly distributed in two sublattices in the following proportions: $\left(\mathrm{Fe}_{0.9} \mathrm{Co}_{0.1}\right)\left(\mathrm{Fe}_{0.1} \mathrm{Co}_{0.9}\right)$, the NL-CPA results for random alloy with bcc structure (opened circles) and the NL-CPA results for the the system with short-range order within the first-neighbor shell (opened diamonds). The dashed line represents the DOS at the Fermi energy, $E_{F}$, as a function of Co concentration. (b) spin resolved DOS for bcc $\mathrm{Fe}_{1-x} \mathrm{Co}_{x}$ for $x=0.01$ (dashed line) and $x=0.5$ (solid line).

the ferromagnetic $3 d$-transition-metal alloy systems bcc $\mathrm{Fe}_{1-x} \mathrm{Co}_{x}$, fcc $\mathrm{Ni}_{1-x} \mathrm{Fe}_{x}$, fcc $\mathrm{Ni}_{1-x} \mathrm{Co}_{x}$ and bcc $\mathrm{Fe}_{1-x} \mathrm{~V}_{x}$.

Fig. 5(a) shows as an example results for the Gilbert damping parameter $\alpha(x)$ calculated for bcc $\mathrm{Fe}_{1-x} \mathrm{Co}_{x}$ for $T=0 \mathrm{~K}$ at different conditions. Full circles represent the results of the single-cite CPA calculations characterizing the random Fe-Co alloy. These results are compared to those obtained employing the non-local $\mathrm{CPA}^{52,53}$ (NL-CPA) assuming no short-range order in the system (opened circles). Dealing in both cases (CPA and NL-CPA), with completely disordered system, the NL-CPA maps the alloy problem on that of an impurity cluster embedded in a translational invariant effective medium determined selfconsistently, thereby accounting

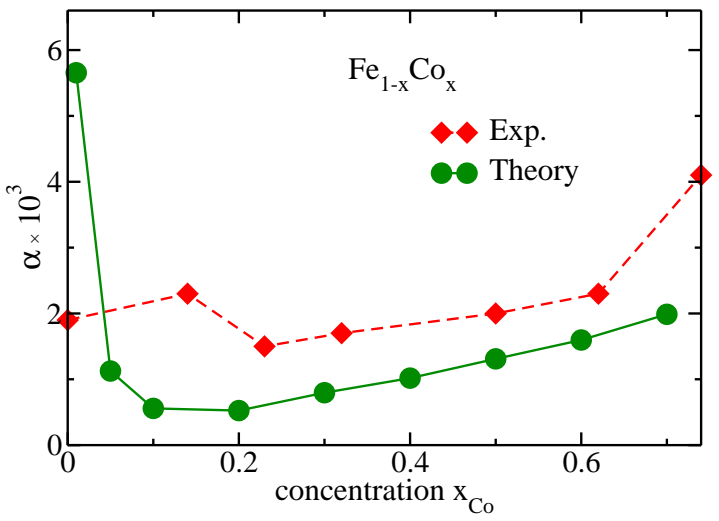

(a)
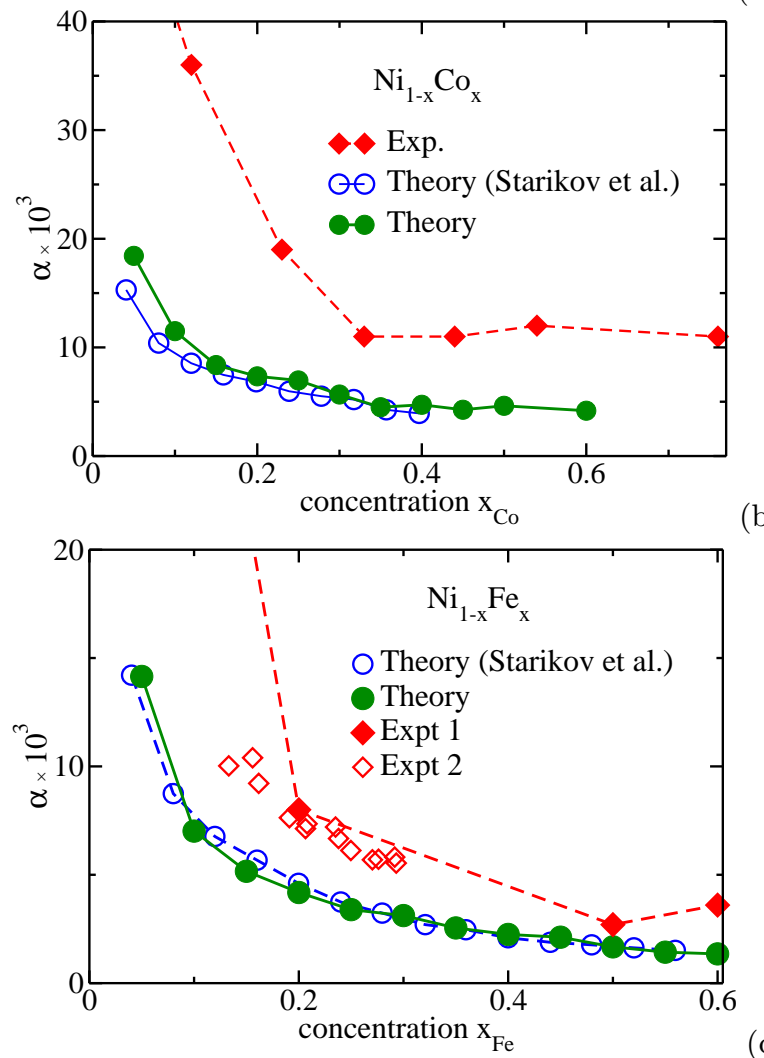

(b)

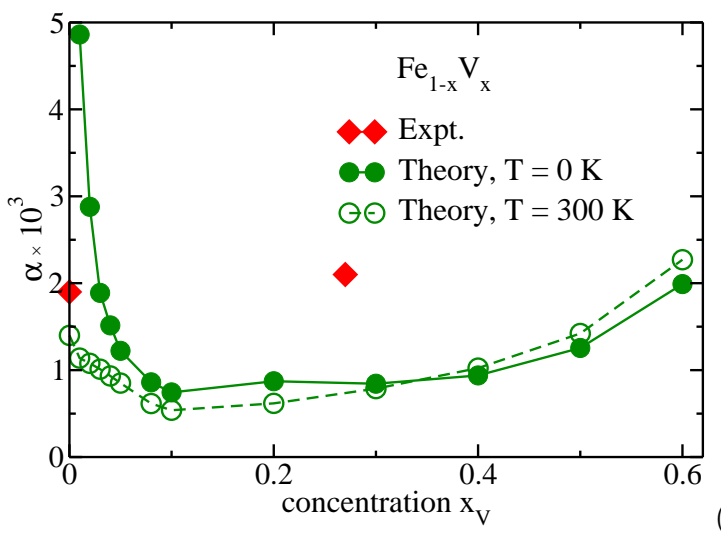

(c)

FIG. 6. The Gilbert damping parameter for $\mathrm{Fe}_{1-x} \mathrm{Co}_{x}$ (a) $\mathrm{Ni}_{1-x} \mathrm{Co}_{x}$ (b) and $\mathrm{Ni}_{1-x} \mathrm{Fe}_{x}$ (c) as a function of Co and $\mathrm{Fe}$ concentration, respectively: present results within CPA (full circles), experimental data by Oogane ${ }^{50}$ (full diamonds). (d) Results for bcc $\mathrm{Fe}_{1-x} \mathrm{~V}_{x}$ as a function of $\mathrm{V}$ concentration: $T=0 \mathrm{~K}$ (full circles) and $T=300 \mathrm{~K}$ (open circles). Squares: experimental data ${ }^{51}$. Open circles: theoretical results by Starikov et al. ${ }^{5}$. 
for nonlocal correlations up to the range of the cluster size. The present calculations have been performed for the smallest NL-CPA clusters containing two sites for bcc based system, accounting for the short-range order in the first-neighbor shell. As one can see, this results in a small decrease of the $\alpha$ value in the region of concentrations around $x=0.5$ (opened diamonds), that is in agreement with the results obtained for partially ordered system (opened square) for $x=0.5$. The latter have been calculated for the $\mathrm{Fe}_{1-x} \mathrm{Co}_{x}$ alloy having $\mathrm{CsCl}$ structure and alloy components randomly distributed in two sublattices in the following proportions: $\left(\mathrm{Fe}_{0.9} \mathrm{Co}_{0.1}\right)\left(\mathrm{Fe}_{0.1} \mathrm{Co}_{0.9}\right)$.

Because the moments and spin-orbit coupling strength do not differ very much for $\mathrm{Fe}$ and $\mathrm{Co}$, the variation of $\alpha(x)$ should be determined in the concentrated regime essentially by the electronic structure at the Fermi energy $E_{F}$. As Fig. 5(a) shows, there is indeed a close correlation with the density of states $n\left(E_{F}\right)$ that may be seen as a measure for the number of available relaxation channels. The change of $\alpha(x)$ due to the increase of the Co concentration is primarily determined by an apparent shift of the Fermi energy also varying with concentration (Fig. 5(b)). The alloy systems considered have the common feature that the concentration dependence of $\alpha$ is governed by the concentration dependent density of states $n\left(E_{F}\right)$.

A comparison of theoretical $\alpha$ values with the experiment for bcc $\mathrm{Fe}_{1-x} \mathrm{Co}_{x}$ is shown in Fig. 6(a), demonstrating satisfying agreement. In the case of $\mathrm{Ni}_{1-x} \mathrm{Fe}_{x}$ and $\mathrm{Ni}_{1-x} \mathrm{Co}_{x}$ alloys shown in Fig. 6, (b) and (c), the Gilbert damping decreases monotonously with the increase of the Fe and Co concentration, in line with experimental data. At all concentrations the experimental results are underestimated by theory approximately by a factor of 2 . The calculated damping parameter $\alpha(x)$ is found in very good agreement with the results based on the scattering theory approach ${ }^{5}$ demonstrating numerically the equivalence of the two approaches. An indispensable requirement to achieve this agreement is to include the vertex corrections mentioned above. As suggested by Eq. (12) the variation of $\alpha(x)$ with concentration $x$ may also reflect to some extent the variation of the average magnetic moment of the alloy, $\mu_{\text {tot }}$.

The peculiarity of the $\mathrm{Fe}_{1-x} \mathrm{~V}_{x}$ alloy when compared to those discussed above is that $\mathrm{V}$ is a non-magnetic metal and has only an induced spin magnetic moment. Despite that, the concentration dependence of the Gilbert damping parameter at $T=0 \mathrm{~K}$ for small amounts of V (see Fig. 6(d)) displays the same trend as the previously discussed alloys shown in Fig. 6(a)-(c). Taking into account a finite temperature of $T=300 \mathrm{~K}$ changes $\alpha$ value significantly at small $\mathrm{V}$ concentrations leading to an improved agreement with experiment for pure $\mathrm{Fe}$, while it still compares poorly with the experimental data at $x_{V}=0.27$. One should stress once more that the concentration dependent behavior of the Gilbert damping parameter of the alloys discussed above is different for an increased amount of impurities (more than 10\%), as a result of a different variation of the DOS $n\left(E_{F}\right)$ caused by a concentration dependent modification of the electronic states and shift of the Fermi level.

\section{C. $5 d$ impurities in $3 d$ transition metals}

As discussed in our recent work $^{28}$ investigating the temperature dependent Gilbert damping parameter for pure $\mathrm{Ni}$ and for $\mathrm{Ni}$ with $\mathrm{Cu}$ impurities, $\alpha$ is primarily determined by the thermal displacement in the regime of small impurity concentrations. This behavior can also be seen in Fig. 7, where the results for Fe with $5 d$-impurities are shown. Solid lines represent results for $T=0 \mathrm{~K}$ for an impurity concentration of 1\% (full squares) and 5\% (full circles). As one can see, at smaller concentrations the maximum of the Gilbert damping parameter occurs for Pt. With increasing impurity content the $\alpha$ parameter decreases in such a way that at the concentration of $5 \%$ a maximum is observed for Os.

The reason for this behavior lies in the rather weak scattering efficiency of $\mathrm{Pt}$ atoms due to a small DOS $n\left(E_{F}\right)$ of the Pt states when compared for example for Os impurities (see Fig. 9). This results in a slow decrease of $\alpha$ at small $\mathrm{Pt}$ concentration when the BFS mechanism is mostly responsible for the energy dissipation. A consequence of this feature can be seen in the temperature dependence of $\alpha$ ( $T=300 \mathrm{~K}$, opened squares): a most pronounced temperature induced decrease of the $\alpha$ value is observed for $\mathrm{Pt}$ and $\mathrm{Au}$. When the concentration of $5 d$-impurities is increased up to $5 \%$, the maximum in $\alpha$ occurs for the element with the most efficient scattering potential resulting in spin-flip scattering processes responsible for dissipation. The temperature effect at this concentration is very small.

Considering in more detail the temperature dependent behavior of the Gilbert damping parameter for Fe with Os and Pt impurities, shown in Fig. 8, one can also observe the consequence of the features mentioned above. At 1\% of Pt impurities $\alpha$ decreases much steeper upon increasing the temperature, as compared to the case of Os impurities. Therefore, in the first case the role of the scattering processes due to atomic displacements is much more pronounced than in the second case with rather strong scattering on the Os impurities. When the concentration increases to $5 \%$ the dependence of $\alpha$ on the temperature in both cases becomes less pronounced.

The previous results can be compared to the results for the $5 d$-impurities in the permalloy $\mathrm{Fe}_{80} \mathrm{Ni}_{20}(\mathrm{Py})$, which has been investigated also experimentally ${ }^{54}$. This system shows some difference in the concentration dependence when compared with pure $\mathrm{Fe}$, because $\mathrm{Py}$ is a disordered alloy with a finite value of the $\alpha$ parameter. Therefore, a substitution of $5 d$ impurities leads to a nearly linear increase of the Gilbert damping with impurity content, just as seen in experiment ${ }^{54}$.

The total damping for $10 \%$ of $5 d$-impurities shown in Fig. 10(a) varies roughly parabolically over the $5 d$ TM se- 


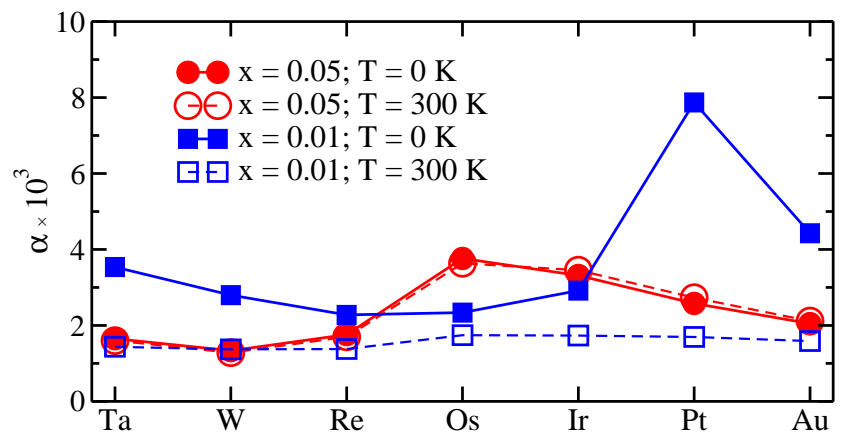

FIG. 7. Gilbert damping parameter for bcc Fe with $1 \%$ (squares) and $5 \%$ (circles) of $5 d$ impurities calculated for $T=0 \mathrm{~K}$ (full symbols) and for $T=300 \mathrm{~K}$ (opened sysmbols).

ries. This variation of $\alpha$ with the type of impurity correlates well with the density of states $n^{5 d}\left(E_{F}\right)$ (Fig. 10(b)). Again the trend of the experimental data is well reproduced by the calculated values that are however somewhat too low.

\section{SUMMARY}

In summary, a formulation for the Gilbert damping parameter $\alpha$ in terms of linear response theory was derived that led to a Kubo-Greenwood-like equation. The scheme was implemented using the fully relativistic KKR band structure method in combination with the CPA alloy theory. This allows to account for various types of scattering mechanisms in a parameter-free way, that might be either due to chemical disorder or to temperatureinduced structural disorder (i.e. electron-phonon scattering effect). The latter has been described by using the so-called alloy-analogy model with the thermal displacement of atoms dealt with in a quasi-static manner. Corresponding applications to pure metals (Fe, Co, Ni) as well as to disordered transition-metal alloys led to very good agreement with results based on the scattering theory approach of Brataas et al. ${ }^{4}$ and well reproduces the experimental results. The crucial role of vertex corrections for the Gilbert damping is demonstrated both in the case of chemical as well as structural disorder and the accuracy of finite-temperature results is analyzed via test calculations.

Furthermore, the flexibility and numerical efficiency of the present scheme was demonstrated by a study on metallic systems on a series of binary $3 d$-alloys $\left(\mathrm{Fe}_{1-x} \mathrm{Co}_{x}, \mathrm{Ni}_{1-x} \mathrm{Fe}_{x}, \mathrm{Ni}_{1-x} \mathrm{Co}_{x}\right.$ and $\left.\mathrm{Fe}_{1-x} \mathrm{~V}_{x}\right), 3 d-5 d$ TM systems, the permalloy-5d TM systems. The agreement between the present theoretical and experimental results is quite satisfying, although one has to stress a systematic underestimation of the Gilbert damping by the numerical results. This disagreement could be caused either by the idealized system considered theoretically (e.g., the boundary effects are not accounted for

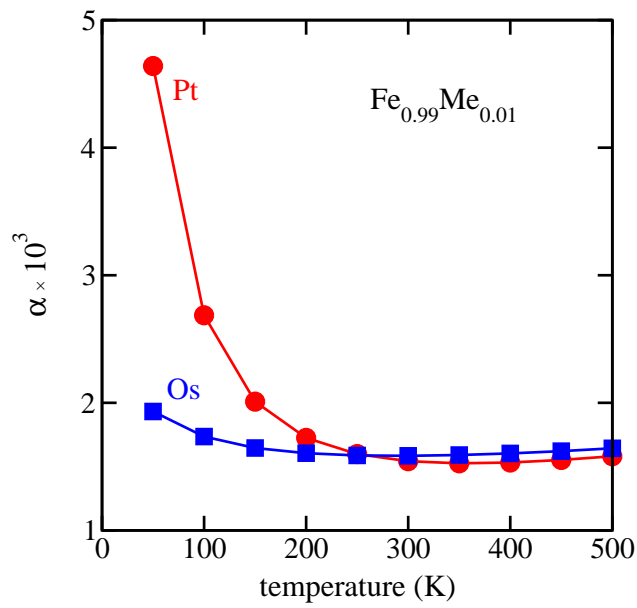

(a)

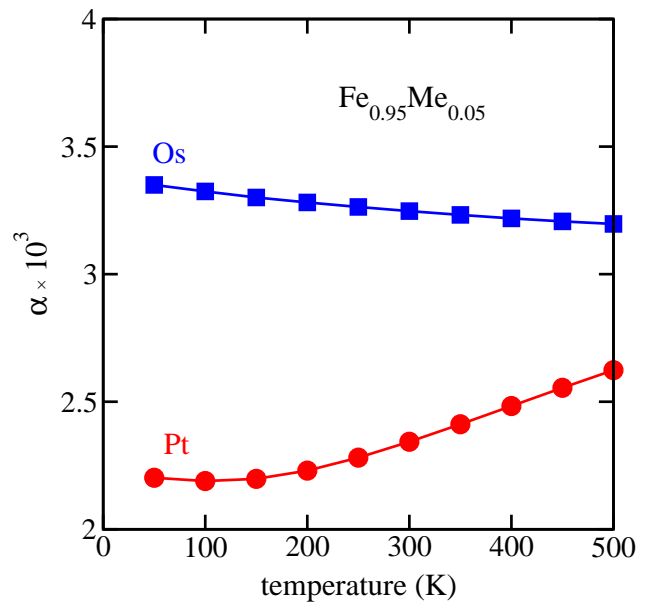

(b)

FIG. 8. Gilbert damping parameter for bcc $\mathrm{Fe}_{1-x} M_{x}$ with $M=\mathrm{Pt}$ (circles) and $M=\mathrm{Os}$ (squares) impurities as a function of temperature for $1 \%$ (a) and $5 \%$ (b) of the impurities.

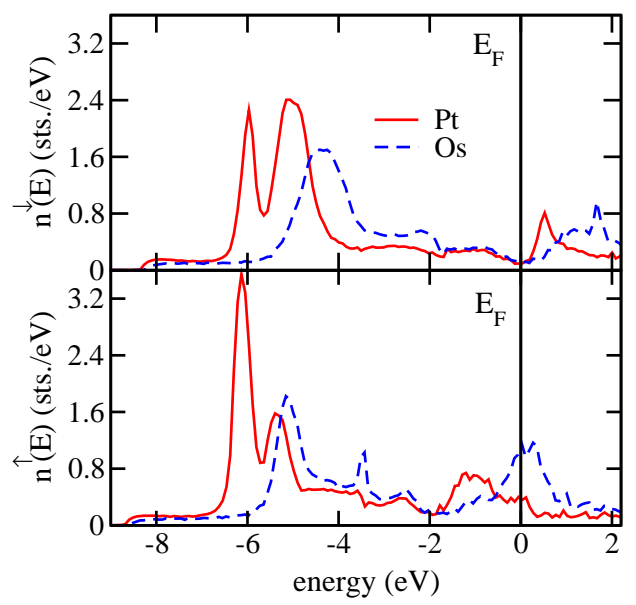

FIG. 9. DOS for $\mathrm{Pt}$ in $\mathrm{Fe}_{1-x} \mathrm{Pt}_{x}$ (full line) and Os in $\mathrm{Fe}_{1-x} \mathrm{Os}_{x}$ (dashed line) for $x=0.01$. 


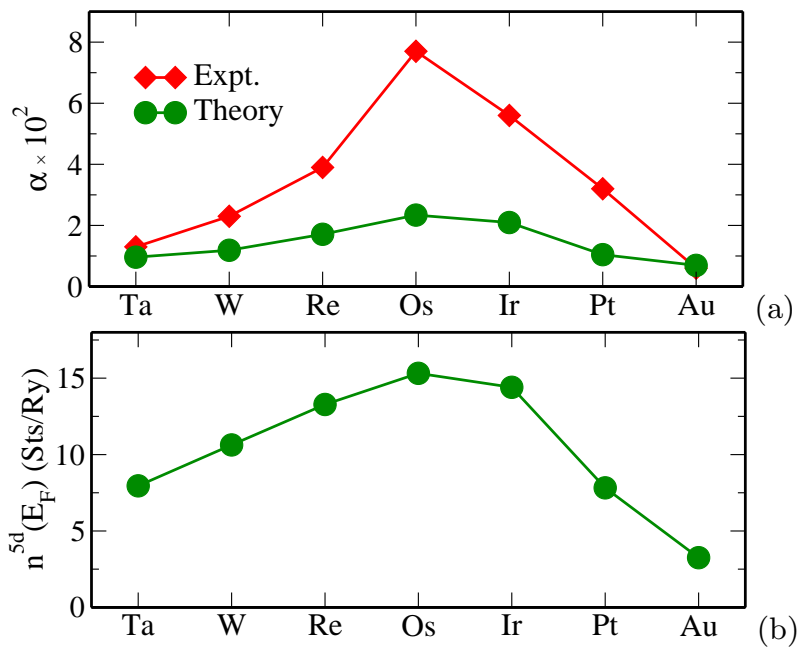

FIG. 10. (a) Gilbert damping parameter $\alpha$ for Py/5d TM systems with $10 \% 5 \mathrm{~d}$ TM content in comparison with experiment ${ }^{54}$; (b) spin magnetic moment $m_{\text {spin }}^{5 d}$ and density of states $n\left(E_{F}\right)$ at the Fermi energy of the $5 d$ component in Py/5d TM systems with $10 \%$ 5d TM content. in present calculations) or because of additional intrinsic dissipation mechanisms for bulk systems which have to be taken into account. These could be, for instance, effects of temperature induced spin disorder ${ }^{44}$.

\section{ACKNOWLEDGMENTS}

The authors would like to thank the DFG for financial support within the SFB 689 "Spinphänomene in reduzierten Dimensionen" and within project EBE-154/23 for financial support.
1 L. Landau and E. Lifshits, Phys. Z. Sovjet. 8, 153 (1935)

2 T. L. Gilbert, IEEE Transactions on Magnetics 40, 3443 (2004)

3 K. Gilmore, Y. U. Idzerda, and M. D. Stiles, Phys. Rev. Lett. 99, 027204 (Jul 2007), http://link.aps.org/doi/ 10.1103/PhysRevLett.99.027204

4 A. Brataas, Y. Tserkovnyak, and G. E. W. Bauer, Phys. Rev. Lett. 101, 037207 (Jul 2008), http://link.aps .org/ doi/10.1103/PhysRevLett.101.037207

5 A. A. Starikov, P. J. Kelly, A. Brataas, Y. Tserkovnyak, and G. E. W. Bauer, Phys. Rev. Lett. 105, 236601 (Dec 2010), http://link.aps.org/doi/10.1103/ PhysRevLett.105.236601

6 V. L. Safonov, J. Appl. Phys. 91, 8653 (2002), http:// link. aip.org/link/?JAP/91/8653/1

7 W. S. Ament and G. T. Rado, Phys. Rev. 97, 1558 (1955), http://link.aps.org/doi/10.1103/PhysRev.97.1558

8 M. Jirsa, phys. stat. sol. (b) 113, 679 (1982)

${ }^{9}$ H. Suhl, IEEE Transactions on Magnetics 34, 1834 (1998)

10 B. Heinrich, R. Urban, and G. Woltersdorf, IEEE Transactions on Magnetics 38, 2496 (2002)

11 R. Arias and D. L. Mills, Phys. Rev. B 60, 7395 (1999), http://link.aps.org/doi/10.1103/PhysRevB.60.7395

12 V. Kambersky and C. E. Patton, Phys. Rev. B 11, 2668 (1975), http://link.aps.org/doi/10.1103/ PhysRevB.11.2668

13 V. Korenman and R. E. Prange, Phys. Rev. B 6, 2769 (1972), http://link.aps.org/doi/10.1103/PhysRevB.6. 2769

14 R. D. McMichael, D. J. Twisselmann, and A. Kunz, Phys. Rev. Lett. 90, 227601 (2003), http://link.aps.org/doi/ 10.1103/PhysRevLett.90.227601

15 Y. Tserkovnyak, A. Brataas, G. E. W. Bauer, and B. I. Halperin, Rev. Mod. Phys. 77, 1375 (2005), http://link. aps.org/doi/10.1103/RevModPhys.77.1375
16 A. Brataas, Y. Tserkovnyak, and G. E. W. Bauer, Phys. Rev. B 84, 054416 (2011), http://link.aps.org/doi/10. 1103/PhysRevB.84.054416

17 M. A. L. Marques, C. A. Ullrich, F. Nogueira, A. Rubio, K. Burke, and E. K. U. Gross, eds., "Time-dependent density functional theory," (Springer-Verlag, Berlin, 2006)

18 M. Fähnle and C. Illg, J. Phys.: Cond. Mat. 23, 493201 (2011), http://stacks.iop.org/0953-8984/23/i=49/a= 493201

19 S. Bhattacharjee, L. Nordström, and J. Fransson, Phys. Rev. Lett. 108, 057204 (Jan 2012), http://link.aps.org/ doi/10.1103/PhysRevLett.108.057204

20 B. Heinrich, D. Fraitova, and V. Kambersky, phys. stat. sol. (b) 23, 501 (1967)

21 Y. Tserkovnyak, G. A. Fiete, and B. I. Halperin, Appl. Physics Lett. 84, 5234 (2004), http://link.aip.org/ link/?APL/84/5234/1

22 S. Zhang and Z. Li, Phys. Rev. Lett. 93, 127204 (2004), http://link.aps.org/doi/10.1103/PhysRevLett.93. 127204

23 M. Fähnle, R. Singer, D. Steiauf, and V. P. Antropov, Phys. Rev. B 73, 172408 (2006), http://link.aps.org/ doi/10.1103/PhysRevB.73.172408

${ }^{24}$ H. J. Skadsem, Y. Tserkovnyak, A. Brataas, and G. E. W. Bauer, Phys. Rev. B 75, 094416 (2007), http://link.aps. org/doi/10.1103/PhysRevB.75.094416

25 V. Kambersky, Can. J. Phys. 48, 2906 (1970)

26 M. Fähnle and D. Steiauf, Phys. Rev. B 73, 184427 (May 2006)

27 V. Kambersky, Czech. J. Phys. 26, 1366 (1976), http:// dx.doi.org/10.1007/BF01587621

28 H. Ebert, S. Mankovsky, D. Ködderitzsch, and P. J. Kelly, Phys. Rev. Lett. 107, 066603 (Aug 2011), http://arxiv.org/abs/1102.4551v1, http: //link.aps.org/doi/10.1103/PhysRevLett.107.066603 
29 W. H. Butler, Phys. Rev. B 31, 3260 (Mar 1985), http: //link.aps.org/doi/10.1103/PhysRevB.31.3260

${ }^{30}$ H. Ebert, in Electronic Structure and Physical Properties of Solids, Lecture Notes in Physics, Vol. 535, edited by H. Dreyssé (Springer, Berlin, 2000) p. 191

31 M. E. Rose, Relativistic Electron Theory (Wiley, New York, 1961) http://openlibrary.org/works/OL3517103W/ Relativistic_electron_theory

${ }^{32}$ H. Ebert and S. Mankovsky, Phys. Rev. B 79, 045209 (2009), http://link.aps.org/doi/10.1103/PhysRevB. 79.045209

33 V. Kamberský, Phys. Rev. B 76, 134416 (Oct 2007), http: //link.aps.org/doi/10.1103/PhysRevB.76.134416

34 E. M. Hankiewicz, G. Vignale, and Y. Tserkovnyak, Phys. Rev. B 75, 174434 (2007), http://link.aps.org/doi/10. 1103/PhysRevB.75.174434

35 I. Garate, K. Gilmore, M. D. Stiles, and A. H. MacDonald, Phys. Rev. B 79, 104416 (2009), http://link.aps.org/ doi/10.1103/PhysRevB.79.104416

36 J. P. Nibarger, R. Lopusnik, Z. Celinski, and T. J. Silva, Appl. Physics Lett. 83, 93 (2003), http://link.aip.org/ link/?APL/83/93/1

37 N. Papanikolaou, R. Zeller, P. H. Dederichs, and N. Stefanou, Phys. Rev. B 55, 4157 (Feb 1997), http://link. aps.org/doi/10.1103/PhysRevB .55.4157

38 A. Lodder, J. Phys. F: Met. Phys. 6, 1885 (1976), http: //stacks.iop.org/0305-4608/6/i=10/a=018

39 N. Stefanou, P. J. Braspenning, R. Zeller, and P. H. Dederichs, Phys. Rev. B 36, 6372 (Oct 1987), http://link. aps.org/doi/10.1103/PhysRevB.36.6372

40 E. M. Gololobov, E. L. Mager, Z. V. Mezhevich, and L. K. Pan, phys. stat. sol. (b) 119, K139 (1983)

41 W. H. Butler and G. M. Stocks, Phys. Rev. B 29, 4217 (Apr 1984), http://link.aps.org/doi/10.1103/ PhysRevB. 29.4217

42 H. Ebert, H. Freyer, A. Vernes, and G.-Y. Guo, Phys. Rev.
B 53, 7721 (Mar 1996), http://link.aps.org/doi/10. 1103/PhysRevB.53.7721

43 V. Kambersky, Czech. J. Phys. 34, 1111 (1984), http:// dx.doi.org/10.1007/BF01590106

44 Y. Liu, A. A. Starikov, Z. Yuan, and P. J. Kelly, Phys. Rev. B 84, 014412 (Jul 2011), http://link.aps.org/doi/10. 1103/PhysRevB.84.014412

45 S. M. Bhagat and P. Lubitz, Phys. Rev. B 10, 179 (1974)

46 B. Heinrich and Z. Frait, phys. stat. sol. (b) 16, K11 (1966), http://dx.doi.org/10.1002/pssb.19660160138

47 G. Woltersdorf, Privat communication

48 D. Steiauf and M. Fähnle, Phys. Rev. B 72, 064450 (Aug 2005), http://link.aps.org/doi/10.1103/PhysRevB. 72.064450

49 C. Gutt, S. Streit-Nierobisch, L.-M. Stadler, B. Pfau, C. M. Günther, R. Könnecke, R. Frömter, A. Kobs, D. Stickler, H. P. Oepen, R. R. Fäustlin, R. Treusch, J. Feldhaus, E. Weckert, I. A. Vartanyants, M. Grunze, A. Rosenhahn, T. Wilhein, S. Eisebitt, and G. Grübel, Phys. Rev. B 81, 100401 (Mar 2010), http://link.aps.org/doi/10.1103/ PhysRevB.81.100401

50 M. Oogane, T. Wakitani, S. Yakata, R. Yilgin, Y. Ando, A. Sakuma, and T. Miyazaki, Jap. J. Appl. Phys. 45, 3889 (2006)

51 C. Scheck, L. Cheng, I. Barsukov, Z. Frait, and W. E. Bailey, Phys. Rev. Lett. 98, 117601 (2007), http://link. aps.org/doi/10.1103/PhysRevLett.98.117601

52 D. A. Rowlands, J. B. Staunton, and B. L. Györffy, Phys. Rev. B 67, 115109 (Mar 2003), http://link.aps.org/ doi/10.1103/PhysRevB.67.115109

53 D. Ködderitzsch, H. Ebert, D. A. Rowlands, and A. Ernst, New Journal of Physics 9, 81 (2007), http://dx.doi.org/ $10.1088 / 1367-2630 / 9 / 4 / 081$

54 J. O. Rantschler, R. D. McMichael, A. Castillo, A. J. Shapiro, W. F. Egelhoff, B. B. Maranville, D. Pulugurtha, A. P. Chen, and L. M. Connors, J. Appl. Physics 101, 033911 (2007) 